\title{
Endoscopic extraction of a metal teaspoon after 12 days of ingestion
}

\section{Daniel Tuculanu* and Dorina-Monica Pârva}

Țuculanu Medical Center, Department of Gastroenterology, Timisoara, Romania

\section{Introduction}

Annually, the objects that come through various ways (involuntarily, voluntarily or post-therapeutically) in the upper (esophagus, stomach, even duodenum) or lower (rectum, colon) digestive tract are more and more various. In parallel, the range of foreign bodies which can be extracted endoscopically was continuously expanded so that increasingly fewer cases are resolved through surgery.

\section{Case}

We present the case of a young woman who swallowed a metal teaspoon (length $14 \mathrm{~cm}$ ) at a party attended by 12 days before the endoscopic intervention extraction.

After 5 days, the patient makes another gastrointestinal endoscopy that confirms the teaspoon in the stomach but cannot remove the foreign body and it is recommended the surgery.

After 12 days of the accident, it was found in the non-contrast radiological examination that the object was still present in the stomach projection area (Figure 1). The patient repeated gastroscopy in our department and we found a teaspoon in stomach, tail oriented towards fornix and the cup in pyloric antrum (Figure 2). The area of the teaspoon was completely corroded but stood out more excoriation and bruising. Nearby was also found a polyp (Figure 3). Initially, we tried to mobilize the teaspoon with a long alligator jaws, but being very

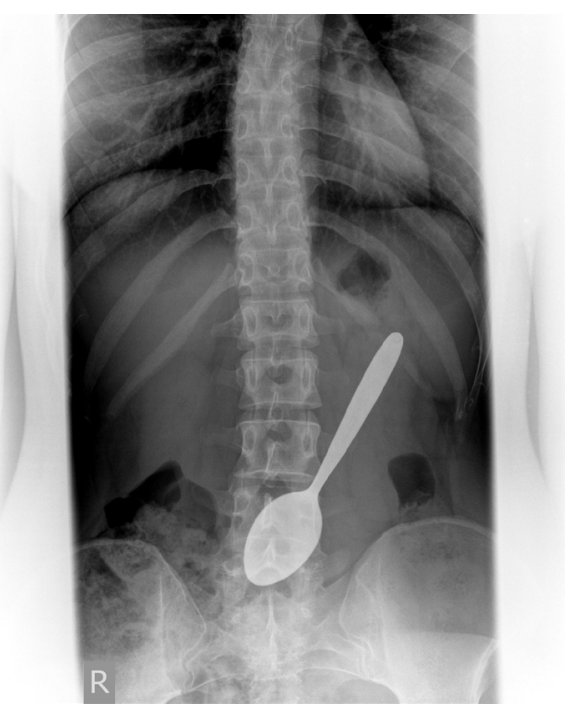

Figure 1. The radiological examination confirms the presence and position of the swallowed teaspoon

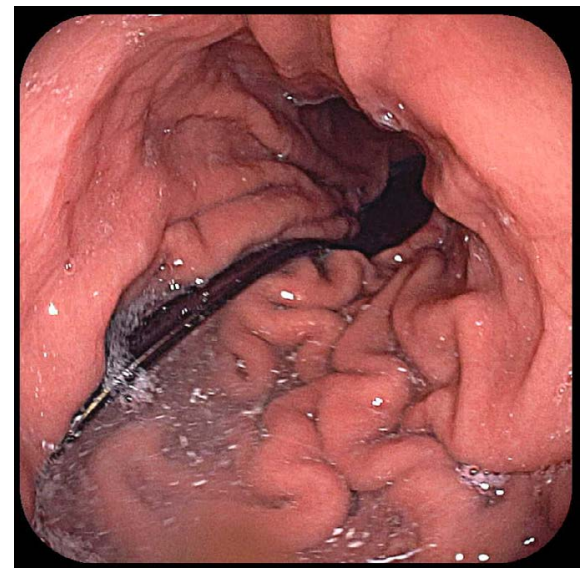

Figure 2. Direction of the teaspoon with the cup to the pyloric antrum and tail to the gastric body



Figure 3. Antral polyp removed with cold polypectomy snare

heavy, we had to capture it with a polypectomy snare (Figure 4). After catching firmly object at the tail using polypectomy loops (Figures 5 \& 6), protected by a latex foreign body protector hood, previously attached to the endoscope tip (Figure 7), drag from the stomach until laryngo-pharynx area where locks and extract digital (Figure 8).

${ }^{\star}$ Correspondence to: Daniel Țuculanu, Țuculanu Medical Center, Department of Gastroenterology, Timisoara, Romania, Tel. 0040-728-955-432; E-mail: danieltuculanu@yahoo.com

Received: May 31, 2018; Accepted: June 11, 2018; Published: June 13, 2018 




Figure 4. Difficult approach to the teaspoon cup with a $35 \mathrm{~mm}$ diameter polypectomy snare

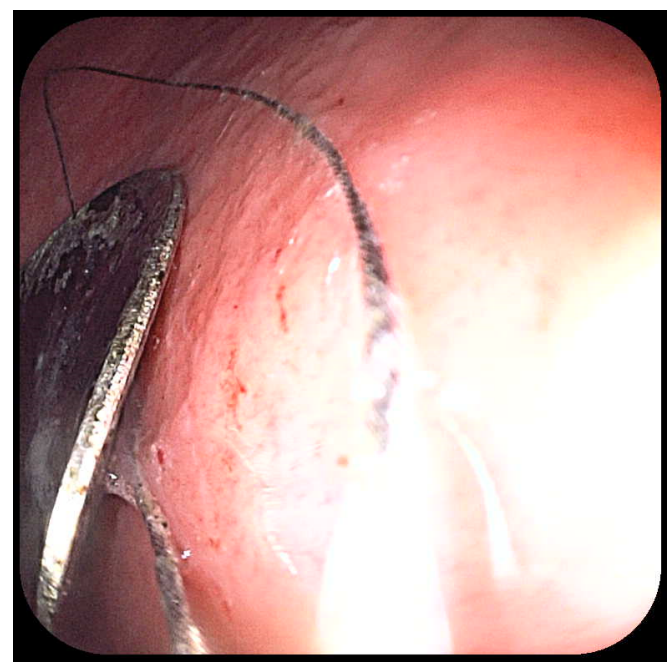

Figure 5. The polypectomy snare redirection to the teaspoon's tail

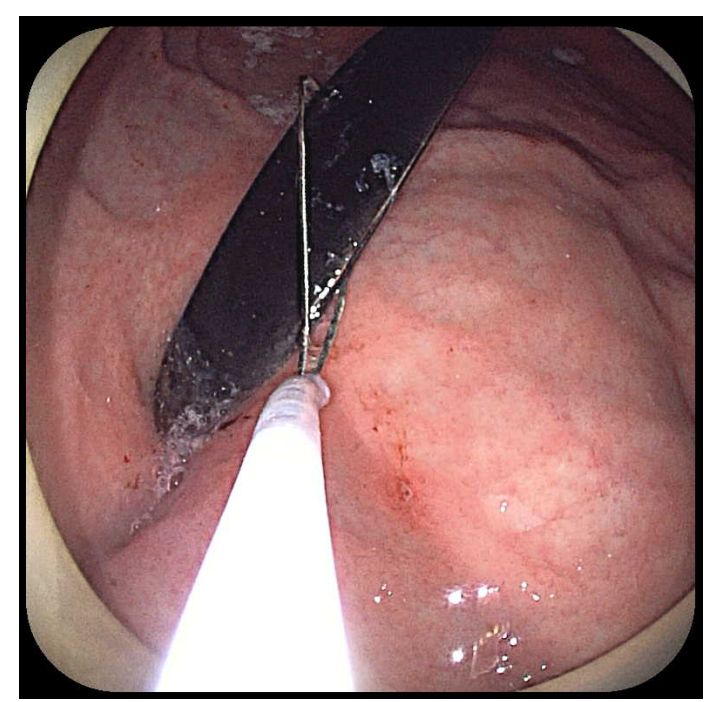

Figure 6. Removing the tail in the hood protector

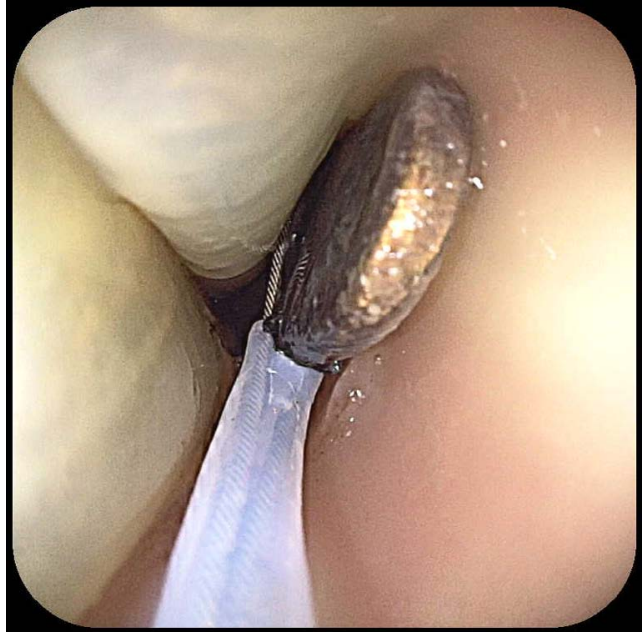

Figure 7. Withdrawal of the polypectomy snare and the endoscope through the esophagus, protected by the latex device

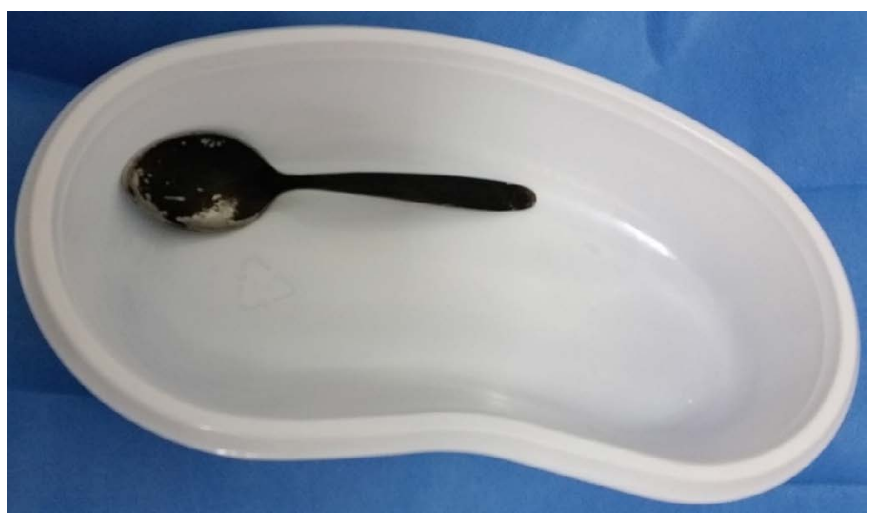

Figure 8. Successful extraction of the foreign body

After extraction were found minor bleeding wounds at the cardia (Figures 9 \& 10).

\section{Discussion}

As the endoscopic equipment and techniques are perfected, the range of foreign bodies extracted from the digestive tract has become increasingly diverse in terms of size, chemical composition, shapes, materials from which they are made and other. The variety of objects penetrated into the digestive tract has led to the imagination of a variety of extraction techniques and extractors. Very often, the endoscopist has to improvise to extract the foreign body from the gastrointestinal tract. This case is interesting because of the size of the swallowed object, which is involuntarily affirmative of a person who has not been confronted with such incidents. As a rule, prisoners swallow similar objects, sometimes even bigger (but after repeated training) to get some benefits. Many of these cases have surgical indication. At this patient, we managed to extract endoscopic without producing significant injuries in the stomach and esophagus. It is also interesting that despite the numerous sharp edges, the teaspoon has not produced stomach lining itself during the 12 days when it stagnated in the stomach and the patient did not show too much discomfort.

In conclusion, digestive endoscopy is a more appropriate therapeutic method for the extraction of digestive foreign bodies. 


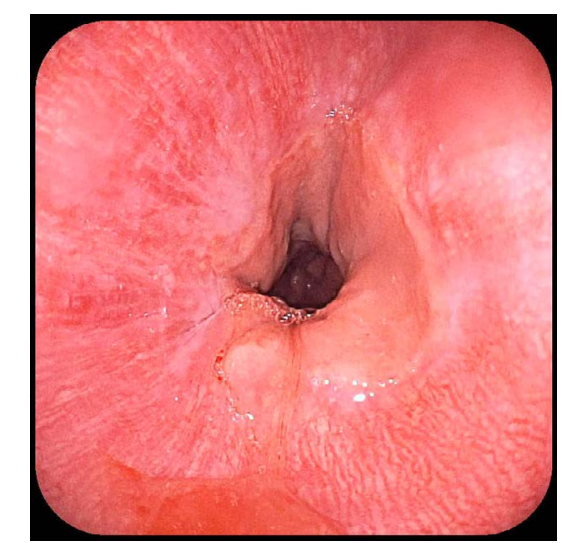

Figure 9. Cardia without significant lesions after procedure

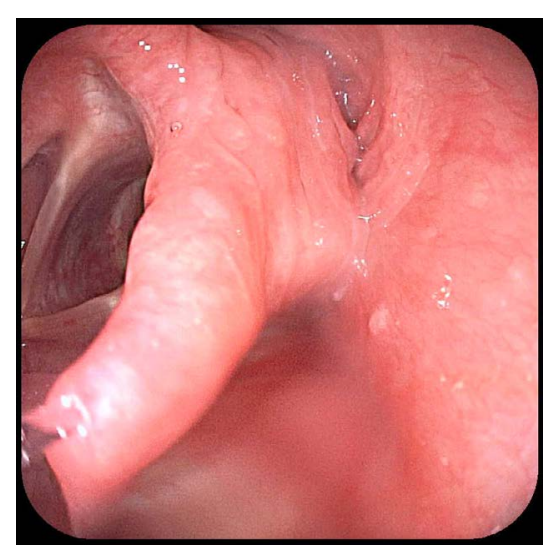

Figure 10. Laryngo-esophageal turn-off without significant lesions after procedure

Copyright: $(02018$ Țuculanu D. This is an open-access article distributed under the terms of the Creative Commons Attribution License, which permits unrestricted use, distribution, and reproduction in any medium, provided the original author and source are credited. 